\title{
Waterproof design for underground engineering of building
}

\author{
Jianfei Sun \\ College of architectural engineering, Heilongjiang University, Harbin 150080, China \\ 2007023@hlju.edu.cn
}

\begin{abstract}
Keywords: construction engineering, underground engineering, waterproof design.
Abstract. In recent years, with the accelerating process of urbanization in China, China's construction industry has gradually entered a period of rapid development. The continuous updating of science and technology has also enabled modern construction technology to be continuously improved, and the quality of construction engineering has also been technically guaranteed. Therefore, it has a high application value for strengthening the underground waterproof construction technology of construction engineering.
\end{abstract}

\section{Introduction}

With the continuous development of the construction industry in China, the utilization of underground building space has been developing in depth, and the quantity and scale of underground engineering construction have increased by a large margin. In the construction process of underground engineering, underground waterproof engineering, as the key link of underground building construction, often play a decisive role in the safety, durability and use function of engineering structures. It can be said that the waterproof quality of underground engineering determines the whole quality and service life of underground engineering. In view of this, the project management enterprises, as the construction of the underground engineering, must adopt professional technical measures and scientific management to ensure the quality of the underground waterproofing engineering, so as to ensure the overall quality of the construction underground engineering.

\section{Causes of underground water seepage in construction Engineering}

Underground waterproof material problem of construction engineering. The construction material is the foundation guarantee of the underground waterproof construction, and the quality of the construction material is decisive to the overall quality of the project. At present, the construction industry continues to develop, the building market is also gradually expanding, and the market competition is increasingly fierce, so the quality of the material in the market is uneven, especially the existence of many cheap, poor quality waterproof materials [1]. If these inferior materials are applied to the underground waterproofing construction, the underground waterproofing performance will be reduced, and water leakage may occur. Moreover, there are some underground waterproofing projects in which the specifications and models of waterproof materials in construction do not conform to the design requirements. Although their performance can meet the requirements of waterproofing to a certain extent, they do not conform to the requirements of the construction projects under construction. This will also make the construction engineering in the use of water-proof problems.

Groundwater environment variation. The stability of underground engineering can be ensured only by artificial treatment of groundwater level in construction area, however, the distribution of groundwater is affected. In addition, the excavation of underground engineering will lead to deformation of soil, which will bring adverse effects to groundwater [2]. As the carrier and medium of groundwater flow, the change of rock and soil structure makes the runoff and recharge of groundwater change, so as to adapt to the change of rock and soil structure, if the change can not continue, there will be leakage, even geological disaster.

Irregularity of construction technology. With the continuous development of construction engineering, the construction technology of building engineering is constantly updated to meet the requirements of the overall level of construction engineering. Because of the low level of construction technology, especially waterproof and impermeable construction technology, many construction 
enterprises can not adapt to the overall requirements of the current construction level, so the application of waterproof and impermeable construction technology in actual construction is not professional enough. For many new waterproof materials, the main points of use are not grasped, and the behavior of blind construction generally exists, which leads to the deterioration of construction quality, and the problem of construction leakage is unavoidable.

Insufficient attention to the underground geological structure or detailed structure of the construction project. In the design of waterproof and impervious system in building engineering, we need to know enough about geological conditions. Because the construction unit does not attach importance to the underground geological structure or the detailed structure of the building project before construction, the construction engineering has not guided the construction according to the appropriate method when dealing with the underground structure. In the process of design, if the geological conditions are not detected carefully, the design of the whole project will be without integrity and standardization [3]. After the project has been put into use for a period of time, the risk of leakage in the building construction project will be greatly enhanced in areas with loose soil. As a result, problems such as construction joints and engineering deformation joints appeared in the project after construction.

\section{Key points of waterproof Design for underground engineering of buildings}

Underground bathroom waterproof design. The quality of the materials must be strictly checked. On the one hand, the construction materials must undergo strict quality inspection before entering the construction site, and the material suppliers must provide the material qualification certificates and quality inspection reports so as to ensure that the material performance parameters meet the design requirements and related standards, for non-conforming materials to be removed. The specification and type of the water supply and drainage pipe should be reasonably determined according to the specific conditions of the construction project, whether the main material or fittings should meet the requirements of the construction design. In construction, the height of toilet is lower than that of other interior space, usually in the $5 \mathrm{~cm}$, do not make the toilet and other interior space height consistent. The concrete guide wall at the bottom of the partition wall of the toilet shall be used as the key point for the construction quality control, and the cement shall be used as the closing operation of asbestos to ensure that the gap between the toilet pipe and the embedded sleeve is filled and dense.

According to the reason of leakage, the innovative idea is put forward. After our continuous research, efforts to analyze the causes of underground leakage, the main reason is that the early design work is not in place, resulting in leakage problems. Specifically, in the early work of the finished product protection measures for the project is not enough. Through a large number of practical studies, it is found that some buildings in our country are very inappropriate for the design of plugging leakage in the course of construction design of residential engineering. And longtime leak plugging project has not been maintained, inevitably there are problems, serious will lead to leakage phenomenon. In addition, there is a specified standard for leak plugging works and the engineering raw materials used in our country, which is also applicable to concrete. But many producers of raw materials in our country are still far from our standards in terms of concrete thickness. The use of this material for construction will certainly cause great harm to our project, if serious, it will be turned into a jerry-built project. In addition, in the process of concrete blending, when and when is usually used to mix, this method is far from the allocation standard of our country. There are also some construction teams that do not pay enough attention to construction projects. When construction problems arise, they often do not solve them in time. This creates a phenomenon of cracks after the end of construction. If serious, there will also be deformation problems. This poses a very serious threat to the life safety and property safety of people. Therefore, we must consider the actual situation of the site during the construction of underground engineering waterproof construction, and then put forward the idea of innovation, the method of innovation, and the innovative design to solve these problems. 
First, the design method should be innovated. In the design process must be linked to the actual situation at that time. And for future maintenance works and repair works should be taken into account, once there are problems, we must promptly solve. In addition, in the process of raw material selection, must be strictly in accordance with China's standards to select, the building more solid and reliable. Do these well, can prevent the occurrence of underground leakage problems to a large extent.

Waterproof Construction Design of Underground Roof. In the waterproof construction of its underground roof, it is necessary to grasp its structure and deal with its base at first. The base at the roof position can be eliminated by means of hard tools, so as to maintain the smoothness of the surface and prevent the wall skin from loosening or bulging. After that, it is necessary to apply the waterproof coating to the position of the subsequent casting strip. When it is smeared, the whole part of its roof needs coating, and the smooth integrity of the coating is ensured. It is also necessary to carry out corresponding waterproof treatment on the slope layer and leveling layer here. In the slope layer, concrete can be used for construction. In the leveling layer, cement mortar can be used for smearing. When waterproofing works are carried out in these two places, it is necessary to select the appropriate position of its thickness, carry on the corresponding construction, and then carry on the projectile line operation on its surface, and mark its elevation control point to carry on the spaced mark. Finally, the activity of the control point is observed, and if the control point is unable to move, the final construction operation can be carried out. Its roof waterproof construction, also need to attach importance to its coil paving, as well as waterproof protective layer construction. In the construction of coiled material, it is necessary to use hot melt method to realize the effect of paving. The hot melt method can make the coiled material and cushion realize effective combination and avoid the situation of water seepage when it is applied. In the construction of protective layer, it is necessary to use fine stone concrete for operation. For the head of the coiled material here, it is necessary to use a block to fix it, and after it has a certain stabilizing effect, plywood is used finally, and the waterproof material here is treated with final protection.

Construction design of 2aterproof of underground exterior wall. In the waterproofing construction of underground wall, it is necessary to understand the structure of each part in detail, and then waterproof construction can be carried out. The first is concrete wall. In the case of waterproof construction here, it is necessary to carry out the setting of the water stop zone according to the construction seam left by the concrete pouring process and the post pouring belt, so as to protect the wall of the wall, and control its slump in a reasonable range. Secondly, reasonable control of the bottom plate and the contact surface of the coiled material should be carried out in waterproof construction. For the vertical wall, as well as the bottom of the coil, it is necessary to contact its here width, to control above $15 \mathrm{~cm}$. At the interface position of the bottom plate coiled material, it is necessary to fix it with the aid of construction equipment, so as to avoid shedding or crimping. Finally, according to the closing of the coiled material. It needs to be pressed, asphalt sealing treatment.

Selection of Curtain Waterproof layer to prevent crack deformation. This method can prevent cracks and deformation to a great extent and make underground waterproof technology more stable [4]. It is mainly external protection against internal affixing. In the process of underground waterproofing construction, one side is smeared with cement mortar, then make napping treatment; the other wall is plucked with polystyrene board, in the process of underground waterproof construction, the plywood is used repeatedly on the other side of the wall. This method can greatly prolong the life of underground waterproofing and greatly reduce the probability of cracks and deformation, which is very beneficial to the household. Therefore, this technology can be vigorously promoted.

Quality measures to control waterproof construction. Problems such as pipeline construction, engineering cracks, bolt structure and so on in the underground base of the building will lead to leakage problems in the underground construction of the building. Therefore, when carrying out the underground waterproofing design of construction projects, these structures should be considered as the key content of the design consideration. At the same time, the quality of waterproof structure should be strictly controlled in construction. For example, in construction of concrete, continuous 
pouring and forming should be adopted as far as possible, and no construction joint should be left as far as possible. If there is a construction joint, it is necessary to choose the reserved position of the construction joint reasonably, choose the position that has less influence on the leakage, and also set up some precipitation joints and post pouring belts when the construction joint is reserved, so as to improve the quality of the waterproof structure and reduce the influence of the construction joint on the underground waterproofing of the construction engineering.

Ensure the rationality of the construction process. It is necessary to do the painting work of the grassroots part. For the base layer, it is necessary to carry out the painting treatment after the acceptance is passed, and at the same time, to ensure the uniformity of the painting work, to avoid the problem of missing brush and the like by thoroughly coating the cold base oil. For the part that has already finished brushing, it is necessary to control the time and make sure that the treatment agent is completely dry before the hot melt method is adopted to carry on the subsequent construction treatment. The hot melt method should be used to lay and paste the coiled material. Before pasting the coiled material, we should do the trial work and ensure the accuracy of dimensions. For the already determined size and coiled material, we should do the cutting work, paste the stubble in time, and use the parallel way to carry on the rolling paste. Under the influence of coiled material heating and base heating, the heating and baking methods should be chosen in the process of using spray lamp. In the process of baking, it is necessary to keep a certain distance between the lamp tip and the coil, and to roll slowly only after the surface is fully melted. When using opposite coiled material for paving, the method of baking and laying should be used. In the process of flat coiled material paving, you can also choose the full-stick method to carry out the construction. In the use of shelves or ladders to lay the coiled material, the top of the use of cloth and so on to wrap to avoid the impact on the already laid coiled material. In the process of flat and facade coils paving, we should use the method of bottom and bottom, and avoid the problem of hollowing and so on.

\section{Conclusions}

In summary, China is still not enough to master the underground waterproof construction technology of construction sites. Therefore, we need to continuously innovate, refer to the leading foreign technology, improve our current technology. Therefore, it is necessary to make preparations before carrying out the design, and then carry out the design on the basis of the concrete investigation of the actual situation to ensure the quality problem. Fundamentally protect the interests of the broad masses of the people and solve practical problems for the people.

\section{References}

[1] Guo Jianxin, Gu Lifang, Quality control measures of underground waterproof construction in building engineering: submitted to Journal of China's High-Tech Enterprise (2016).

[2] Kong Aisan, Study on Construction quality Control measures of Waterproof Engineering in Architectural Engineering: submitted to Journal of Management and Technology of small and Medium-sized Enterprises (2016).

[3] Li Jianzeng, Quality Control and Measure Analysis of Waterproof Construction in Building Engineering: submitted to Journal of Enterprise Technology Development (2017).

[4] Chen Bo, Chen Tao, Strategic research on underground water-proof construction of construction works: submitted to Journal of Farmhouse staff (2017). 